\title{
Opening up the QCD axion window
}

\author{
Prateek Agrawal, ${ }^{a}$ Gustavo Marques-Tavares ${ }^{b}$ and Wei Xue ${ }^{c}$ \\ ${ }^{a}$ Department of Physics, Harvard University, \\ 17 Oxford Street, Cambridge, U.S.A. \\ ${ }^{b}$ Stanford Institute for Theoretical Physics, Stanford University, \\ 382 Via Pueblo Mall, Stanford, U.S.A. \\ ${ }^{c}$ Center for Theoretical Physics, Massachusetts Institute of Technology, \\ 77 Massachusetts Avenue, Cambridge, U.S.A. \\ E-mail: prateekagrawal@fas.harvard.edu, gusmt@stanford.edu, \\ weixue@mit.edu
}

ABSTRACT: We present a new mechanism to deplete the energy density of the QCD axion, making decay constants as high as $f_{a} \simeq 10^{17} \mathrm{GeV}$ viable for generic initial conditions. In our setup, the axion couples to a massless dark photon with a coupling that is moderately stronger than the axion coupling to gluons. Dark photons are produced copiously through a tachyonic instability when the axion field starts oscillating, and an exponential suppression of the axion density can be achieved. For a large part of the parameter space this dark radiation component of the universe can be observable in upcoming CMB experiments. Such dynamical depletion of the axion density ameliorates the isocurvature bound on the scale of inflation. The depletion also amplifies the power spectrum at scales that enter the horizon before particle production begins, potentially leading to axion miniclusters.

KEYwords: Cosmology of Theories beyond the SM, CP violation

ARXIV EPRINT: 1708.05008 


\section{Contents}

1 Introduction 1

2 Axion dark matter review 3

2.1 The axion Lagrangian and potential 4

2.2 Axion cosmology 5

$\begin{array}{lll}3 & \text { A simple model for particle production } & 6\end{array}$

4 Particle production and $\Omega_{\mathrm{DM}} \quad \mathbf{8}$

4.1 The equations of motion 8

$\begin{array}{ll}4.2 \text { Conditions for efficient particle production } & 10\end{array}$

$\begin{array}{lll}4.3 & \text { Dark photon contribution to } N_{\text {eff }} & 11\end{array}$

$\begin{array}{lll}4.4 & \text { Numerical results } & 12\end{array}$

$\begin{array}{llr}5 & \text { Future directions and conclusions } & 15\end{array}$

A UV completions of the alignment model $\quad 16$

$\begin{array}{lll}\text { A.1 A 5D model } & 17\end{array}$

$\begin{array}{lll}\text { A.2 Confining dynamics } & 17\end{array}$

$\begin{array}{lll}\text { A.3 } & \text { Clockwork } & 18\end{array}$

\section{Introduction}

Dark matter remains the most compelling evidence of physics beyond the Standard Model (SM). Since we have only observed it through its gravitational effects, there is a wide range of possible dark matter candidates. A very interesting possibility is that dark matter is made of light bosonic fields which only interact extremely weakly with the SM [1]. The QCD axion is a particularly well-motivated example of such weakly-coupled light fields [2-5] (see [6] for a review). An approximate shift symmetry protects its mass and couplings, so that it can be naturally light and have a lifetime much longer than the age of the universe. An axion field oscillating about the minimum of its potential has the equation of state of matter, making it a dark matter candidate [7-9]. Further, the axion makes the CP-violating QCD $\theta$ angle dynamical, with a minimum at $\bar{\theta}=0[10]$. Thus, the axion can naturally explain the severe constraints on $\mathrm{CP}$ violation in the strong sector, $\bar{\theta} \lesssim 10^{-10}$, from null measurements of the neutron/Hg electric dipole moments [11-13].

The mass of the QCD axion is set by its decay constant $f_{a}$. Its couplings to other SM fields are model-dependent, but are expected to be given by higher-dimension operators suppressed by $f_{a}$. Astrophysical observations impose a lower bound on the decay constant, $f_{a} \gtrsim 10^{9} \mathrm{GeV}$ for generic couplings [14-18] (for detailed discussion of the constraints 
see [6]). On the other hand, axion decay constants of $f_{a}>10^{12} \mathrm{GeV}$ predict a dark matter abundance in excess of observations for generic initial conditions. These two considerations determine the axion window, $10^{9} \mathrm{GeV}<f_{a}<10^{12} \mathrm{GeV}$, within which axions can naturally account for the observed dark matter density. There are a number of ongoing and proposed experiments for probing the QCD axion window [19-23].

We propose a new mechanism for depleting the axion abundance in the early Universe, such that the region $f_{a}>10^{12} \mathrm{GeV}$ becomes viable. This region is well-motivated theoretically. Generic expectations from string theory predict $f_{a}$ values higher than the axion window [24-26]. Beyond theoretical arguments, a practical reason for exploring viable cosmological models outside the axion window is that there are a number of exciting proposed experiments that should have sensitivity to very light (and thus large- $f_{a}$ ) axions [27-32].

Our mechanism uses particle production in the time-dependent axion background. We assume that the axion couples to a massless dark photon, a U(1) gauge boson that is decoupled from the SM. As the axion starts oscillating in the early universe, certain modes of the gauge field become tachyonic, leading to an exponential growth of such modes. A similar mechanism has been previously used for generating primordial magnetic fields [33-39], for inflation and (p)reheating after inflation [40-47], for moduli decay [48], and for the relaxion [49]. Our mechanism differs from previous cases because we focus on smaller couplings of the axion to the gauge fields, which requires multiple oscillations of the axion until the gauge field grow sufficiently to influence the axion dynamics. In this regime the relevant modes of the gauge field go through two separate phases, a tachyonic growth period and a subsequent parametric resonance period. Thus, our mechanism shares features both with tachyonic preheating and with more standard preheating scenarios based on parametric resonant decays.

Even though the coupling responsible for this mechanism is quite generic for axion-like particles, the presence of light charged degrees of freedom can make the production of gauge fields very inefficient due to plasma screening effects. For this reason we focus on the coupling of the axion to a dark photon instead of making use of its couplings to the SM photon.

We show that this mechanism can be very efficient in transferring energy from the axion into the dark photons. As long as backscattering effects of the dark photons into the axions are small, this mechanism can open up most of the large- $f_{a}$ parameter space. We will briefly comment on potential limitations of this mechanism due to the possibility of backscattering effects introducing significant deviations in our results. A quantitative understanding of these effects requires a dedicated numerical investigation beyond the scope of this work.

There are other ways to evade the upper bound on $f_{a}$. One possibility is that the initial misalignment angle is tuned to be small, with the tuning justified by anthropic selection of the dark matter density [50,51]. Another possibility is that the universe experienced entropy production between the QCD phase transition and Big Bang Nucleosynthesis [8, 52-54]. This mechanism can relax the bound to $f_{a} \lesssim 10^{15} \mathrm{GeV}$. If the axion potential was temporarily much larger in the very early universe, such that $m_{a}>H$, it would relax to its minimum earlier [55-58]. However, a viable model that generates a large axion potential is 
typically quite involved and needs to explain why the early time minimum is aligned with the minimum today. There are other possible signatures of our mechanism, which can be potentially used to distinguish it from these other mechanisms.

- For very large $f_{a}$, the energy in the axion is so large that, once it is transferred to radiation, it can lead to observable changes in the number of extra relativistic degrees of freedoms $\left(N_{\text {eff }}\right)$ during $\mathrm{BBN}$ and during the $\mathrm{CMB}$ decoupling. Current constraints on $N_{\text {eff }}$ limit $f_{a} \lesssim 2 \times 10^{17} \mathrm{GeV}$ for an axion with order one initial misalignment angle. Future measurements are expected to improve the bound on $N_{\text {eff }}$ by an order of magnitude which will provide indirect tests of this mechanism in a large range of the interesting parameter space.

- A dynamical mechanism that decreases the axion abundance also significantly weakens the bounds on large- $f_{a}$ axions from the non-observation of isocurvature perturbations in the CMB. This opens up a sizable fraction of the parameter space for inflationary models, specially for higher scales of inflation. If future experiments measure a large scale for inflation and also discover axion DM with a large $f_{a}$, this would be a strong indirect evidence against an anthropic axion scenario.

- The particle production mechanism might also lead to significant changes in the small scale matter power spectrum. This is due to a combination of effects which we describe in the text. One of the main effects is that it decreases the average energy density in the axion field without a corresponding decrease in the energy density of its fluctuations at scales that enter the horizon before particle production becomes relevant. This amplifies the density perturbations generated by inflation and enhances the power spectrum at these small scales.

Even though the most economical possibility is that a single axion field solves the strong-CP problem as well as constitutes all of dark matter, there may be a plenitude of light axions [59], since axions arise readily in string theory compactifications [24-26]. While in this paper we will focus on the QCD axion, our results can be easily adapted to other axion-like particles.

After a brief review of axion dark matter in section 2, we will present a simple model of axions coupled to the dark photon with appropriate strength to produce our effect in section 3. We then describe the mechanism of particle production and our results in section 4 , and we lay out the future directions and conclusions in section 5 .

\section{Axion dark matter review}

The cosmology of the axion depends sensitively on whether the spontaneous breaking of PQ symmetry occurs before/during or after inflation. In this work we are interested in the case where the PQ symmetry is broken before inflation, which is the case when the scale of PQ breaking is larger than both Hubble during inflation and the reheat temperature. Due to the astrophysical constraints requiring $f_{a} \gtrsim 10^{9} \mathrm{GeV}$, and the bound on the inflationary scale from non-detection of gravitational B-modes in the CMB [60,61], $H_{I} \lesssim 10^{14} \mathrm{GeV}$, 
this scenario represents a large fraction of the allowed axion parameter space. In this case, due to the exponential expansion during inflation, the axion field takes on a constant value throughout our Hubble volume, which is generically away from the late time minimum of the potential. The dark matter abundance then arises out of this misalignment, and depends on the axion mass and on the size of the misalignment.

\subsection{The axion Lagrangian and potential}

We review the axion couplings to the SM. Following [62] the relevant terms in the axion Lagrangian are given by

$$
\mathcal{L}=\frac{1}{2}\left(\partial_{\mu} \phi\right)^{2}+\frac{\alpha_{s} \phi}{8 \pi f_{a}} G_{\mu \nu}^{a} \tilde{G}^{a \mu \nu}+g_{a \gamma \gamma} \phi F^{\mu \nu} \tilde{F}_{\mu \nu}+\frac{\partial_{\mu} \phi}{2 f_{a}} \sum_{\psi} c_{\psi}\left(\bar{\psi} \gamma^{\mu} \gamma^{5} \psi\right)
$$

where $\tilde{G}^{\alpha \beta}=\frac{1}{2} G_{\mu \nu} \epsilon^{\mu \nu \alpha \beta}$, and $\psi$ are SM fermions. We use the coupling to gluons as the definition of $f_{a}$, and the couplings $c_{i}$ to other SM fields are model-dependent. The coupling of the axion to photons is relevant for many experimental searches, and for the KSVZ axion $[63,64]$ is given by

$$
g_{a \gamma \gamma}=\frac{-1.92 \alpha}{8 \pi f_{a}} .
$$

The coupling of the axion to QCD breaks the continuous shift symmetry of the axion to a discrete shift symmetry. For the axion to solve the strong-CP problem, it should get its potential dominantly from QCD dynamics. The zero temperature potential is a low energy observable and can be calculated directly from the chiral Lagrangian (see e.g. [62]),

$$
V(\phi)=-m_{\pi}^{2} f_{\pi}^{2} \sqrt{1-\frac{4 m_{u} m_{d}}{\left(m_{u}+m_{d}\right)^{2}} \sin ^{2} \frac{\phi}{2 f_{a}}} \approx-m_{\pi}^{2} f_{\pi}^{2}+\frac{m_{\pi}^{2} f_{\pi}^{2}}{2 f_{a}^{2}} \frac{m_{u} m_{d}}{\left(m_{u}+m_{d}\right)^{2}} \phi^{2}+\mathcal{O}\left(\phi^{4} / f_{a}^{4}\right)
$$

for small $\phi$ displacements.

At high temperature, the axion potential is suppressed. There are many computations of the high temperature axion mass (dilute instanton gas, lattice, instanton liquid model), which do not all agree with each other (see e.g. [65-70] for recent results and discussion). This is an important open question which has implications for the axion dark matter abundance, but which does not change the conclusion that large $f_{a}$ axions generically lead to an overabundance of dark matter. For clarity, we parametrize the mass in the following simple way which resembles the result from the dilute instanton gas calculation [71, 72],

$$
m_{a}(T)= \begin{cases}m_{a}(0), & T<200 \mathrm{MeV} \\ b m_{a}(0)\left(\frac{200 \mathrm{MeV}}{T}\right)^{4}, & T \geq 200 \mathrm{MeV}\end{cases}
$$

where $m_{a}(0)=(78 \mathrm{MeV})^{2} / f_{a}$ and $b=0.018$. Note that in our approximation the mass is discontinuous, which is not expected to be actual behavior for the axion mass. The simple power law dependence in the temperature is expected to hold at large temperatures, $T \gtrsim 1 \mathrm{GeV}$, where the dilute instanton gas approximation is reliable. Given the disagreement in how to estimate the axion potential closer to the QCD phase transition scale, we 
extended the higher temperature behavior past the regime where it is reliable in order to have a concrete formula. As already discussed this introduces uncertainties in the calculation for the axion abundance but does not change the qualitative picture which is what we want to focus on.

\subsection{Axion cosmology}

Axions are produced in the early universe via thermal production through its couplings to the SM plasma [73-75] and non-thermally via the misalignment mechanism [9]. Thermally produced axions have momenta comparable to the temperature of the SM plasma and thus are an extra form of radiation. The abundance of thermal axions is negligible for large $f_{a}$, which is the case we are interested in. The non-thermal production generates a coherent oscillating field, which behaves as a non-relativistic fluid and can be the dominant contribution to the dark matter density. The relic density is determined by the initial misalignment angle of the axion, $\theta_{i}=\phi_{i} / f_{a}$. The axion starts to oscillate when $m_{a} \sim H$, and can be subsequently described as a bath of zero momentum particles. The axion abundance can be easily calculated by using the fact that shortly after oscillations begin the axion co-moving number density is conserved. If $f_{a}>2 \times 10^{17} \mathrm{GeV}$, the axion starts to oscillate at $T_{\mathrm{osc}}<\Lambda_{\mathrm{QCD}} \simeq 200 \mathrm{MeV}$, when the axion mass is roughly constant. For $f_{a}<2 \times 10^{15} \mathrm{GeV}$ the oscillations begin at a higher temperature, $T_{\mathrm{osc}} \gtrsim 1 \mathrm{GeV}$, where the axion mass depends sensitively on the temperature and is well approximated by eq. (2.4). In these two regions, the axion DM relic density is [71],

$$
\Omega_{a} h^{2} \sim\left\{\begin{array}{l}
2 \times 10^{4}\left(\frac{f_{a}}{10^{16} \mathrm{GeV}}\right)^{7 / 6} \theta_{i}^{2}, f_{a}<2 \times 10^{15} \mathrm{GeV} \\
5 \times 10^{3}\left(\frac{f_{a}}{10^{16} \mathrm{GeV}}\right)^{3 / 2} \theta_{i}^{2}, f_{a}>2 \times 10^{17} \mathrm{GeV}
\end{array},\right.
$$

where we have kept only the quadratic piece of the axion potential. In the region $2 \times 10^{15} \mathrm{GeV}<f_{a}<2 \times 10^{17} \mathrm{GeV}$, the axion begins oscillation around the QCD scale, $T_{\mathrm{osc}} \sim \Lambda_{\mathrm{QCD}}$. In this region neither expression above is accurate and strong QCD effects affect the axion abundance. For our numerical results we calculate the axion abundance explicitly by numerically evolving the axion condensate using the temperature-dependent mass in eq. (2.4). Using the estimate above, we see that for $f_{a} \sim 10^{15} \mathrm{GeV}$, we would need an initial misalignment $\theta_{i}^{2} \sim 10^{-4}$ to get the observed relic density.

Another important constraint on axion dark matter comes from the limit on isocurvature perturbations. Because the axion is effectively a free scalar field during inflation, it experiences scale invariant fluctuations due to the de Sitter background $\delta \phi \sim H_{I} /(2 \pi)$. These correspond to isocurvature perturbations and are constrained from CMB measurements. The isocurvature power spectrum due to axion fluctuations is given by

$$
P_{\mathrm{iso}}=\frac{\Omega_{a}^{2}}{\Omega_{\mathrm{DM}}^{2}} \frac{H_{I}^{2}}{\pi^{2} f_{a}^{2} \theta_{i}^{2}},
$$

where we allowed the axion abundance to differ from the observed dark matter abundance $\Omega_{\mathrm{DM}}$. This value depends on the $\theta_{i}$, because the perturbations are defined by $\delta \rho_{a} / \rho_{a}$, and the average density of the axions depends on the misalignment angle. 
The CMB has measured the scalar perturbations as predominantly adiabatic perturbations with a scalar power spectrum, $P_{s} \simeq 2 \times 10^{-9}$, where we neglect the spectral index. With the assumption that the scalar power spectrum is uncorrelated with the axion isocurvature perturbations, CMB constrains [60],

$$
r_{\text {iso }}=P_{\text {iso }} / P_{s}<0.038 \text {. }
$$

The bound on isocurvature perturbations leads to an upper bound on Hubble during inflation

$$
\frac{H_{I}}{f_{a}} \lesssim 10^{-5} \theta_{i} \frac{\Omega_{\mathrm{DM}}}{\Omega_{a}}
$$

If axion constitutes all of dark matter from eq. (2.5) one sees that for large $f_{a}$ one requires $\theta_{i} \ll 1$, which makes the isocurvature bound on the inflationary scale stronger. Therefore if the initial misalignment angle is $\mathcal{O}(1)$, and a dynamical mechanism decreases the axion abundance to the observed DM abundance, the isocurvature bound is weakened. This is the case for the late entropy production scenarios [76, 77], and is also the case for the particle production mechanism we explore in this work. For other recent ideas that relax the axion isocurvature bounds see e.g. [78-80].

There are a number of tabletop experiments and astrophysical observations that put constraints on the viable axion parameter space. There are also new experimental proposals which have the potential to reach the QCD axion in a wide mass window in the near future. The existing limits and future sensitivity on the axion coupling to photons are summarized in figure 1. We highlight the light axion region, which is the region of interest for new experiments. This region would be ruled out for generic initial conditions, but our mechanism makes the entire region viable. The blue and red shaded regions in this figure represents the current bounds. It includes the cavity microwave experiment, ADMX [19], which has probed axion DM of about $\mu \mathrm{eV}$; the helioscope, CAST [14], to search for solar axion coupling to photons; the supernova SN1987A bound [18] from the Primakoff process; FermiLAT [17], H.E.S.S [16] and X-ray observations [81-83] bounds from the irregularity of the photon spectrum; globular clusters [15]; black hole superradiance [28] (ignoring the disruption of the axion cloud due to interactions at larger $\left.g_{a \gamma \gamma}\right)$. We show the projected sensitivity of future experiments, ABRACADABRA [30], ALPS II [84], ADMX [20], CASPEr-Wind and CASPEr-Electric [27], and the helioscope IAXO [21].

\section{A simple model for particle production}

In addition to the standard QCD axion Lagrangian discussed above, we assume an additional coupling of the axion to a dark photon (such couplings have recently been explored in a different context $[85,86])$,

$$
\mathcal{L}=\frac{\alpha_{d} c_{d}}{8 \pi f_{a}} \phi F_{D}^{\mu \nu}\left(\widetilde{F}_{D}\right)_{\mu \nu}
$$

As we will see below, the particle production mechanism is only effective when the coupling of the axion to the dark photon is larger than the coupling to QCD, with $r \equiv \frac{c_{d} \alpha_{d}}{2 \pi} \sim \mathcal{O}(10)$. 


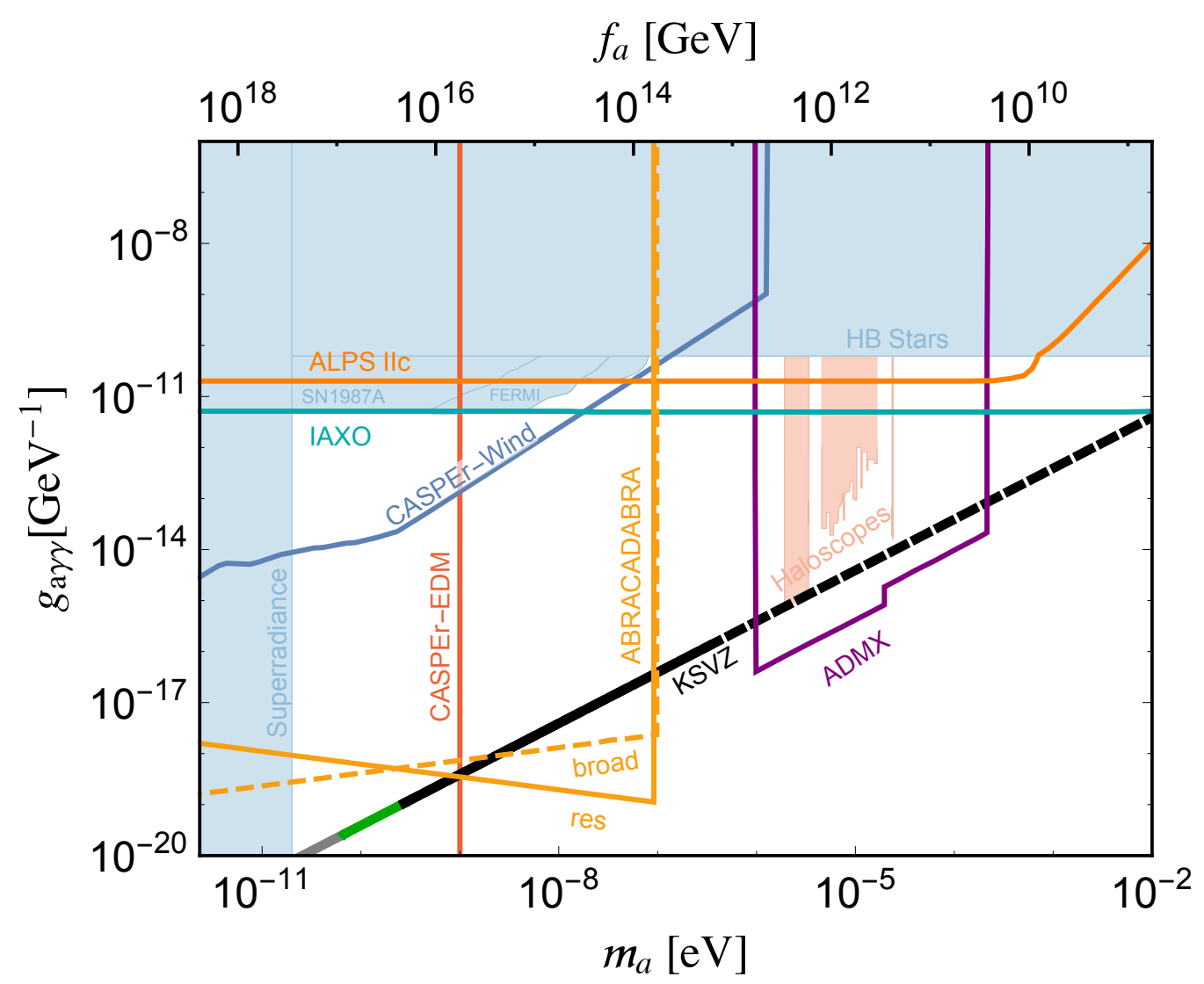

Figure 1. Current bounds and future experimental reach for axion couplings to photons defined in eq. (2.1). Blue shaded regions show the current constraints which are independent of a dark matter interpretation, while the red shaded region are constraints from axion haloscopes. Sensitivity of future experiments is shown by colored lines. The thick black line shows the prediction for the KSVZ axion as in eq. (2.2). The solid part of the line indicates where the initial misalignment would need to be tuned $\theta_{i}<0.1$ or worse, which is where our mechanism is needed to have the correct axion abundance. For larger values of $f_{a}$ (the green part of the line) the extra dark radiation produced by our mechanism will be within the sensitivity of future CMB experiments. Even larger $f_{a}$ values (the gray part), are already constrained by Planck observations (see discussion in section 4.3).

This hierarchy of couplings is easily seen to be stable under quantum corrections at the effective theory level. Nevertheless, one may wonder how easy it is to obtain this structure from a UV model. There are a number of possible model building avenues. We will not pursue them in detail here, but present a simple example as a proof of principle.

One way to generate a hierarchy in axion couplings is using the Kim-Nilles-Peloso (KNP) alignment mechanism [87-96]. As a specific case, we take two axions, say $a$ and $b$, each coupling separately to the two gauge groups, QCD and the dark photon, respectively. We include an additional "aligned" contribution to the axion mass matrix which breaks the two shift-symmetries down to a single shift-symmetry such that one linear combination of $a$ and $b$ gets a large mass and decouples. If the light eigenstate has a hierarchical overlap with $a$ vs. $b$, then it couples to QCD and the dark photon with a hierarchical coupling. 
The effective Lagrangian is,

$$
\mathcal{L}=\frac{\alpha_{s}}{8 \pi f} a G^{a, \mu \nu} \widetilde{G}_{\mu \nu}^{a}+\frac{\alpha_{d}}{8 \pi f} b F_{D}^{\mu \nu}\left(\widetilde{F}_{D}\right)_{\mu \nu}+\Lambda^{4} \cos \left(\frac{n a+b}{f}\right)
$$

where we have included a simple example of the explicit symmetry breaking term that we want. This symmetry breaking term can be UV-completed in a number of different ways, which we discuss in appendix A.

The eigenstates from the above Lagrangian are,

$$
\phi=\frac{1}{\sqrt{1+n^{2}}}(a-n b), \quad \phi_{h}=\frac{1}{\sqrt{1+n^{2}}}(n a+b)
$$

where $\phi$ is the QCD axion, and $\phi_{h}$ is the heavy mass eigenstate. We can thus rewrite the effective Lagrangian in terms of the light eigenstate,

$$
\mathcal{L}=\frac{\alpha_{s}}{8 \pi f_{a}} \phi G^{a, \mu \nu} \widetilde{G}_{\mu \nu}^{a}+\frac{\alpha_{d} n}{8 \pi f_{a}} \phi F_{D}^{\mu \nu}\left(\widetilde{F}_{D}\right)_{\mu \nu}
$$

with $f_{a}=f \sqrt{1+n^{2}}$. In other words, $c_{d}=n$, or $r=n \alpha_{d} /(2 \pi)$.

\section{Particle production and $\Omega_{\mathrm{DM}}$}

We now study the changes in the axion evolution due to its coupling to the dark photon. In our setup, the QCD axion couples to dark photons, with a coupling that is $\sim \mathcal{O}(10)$ times larger than its coupling to the standard model particles. This coupling is responsible for depleting the axion abundance.

If the mass of the dark photon exceeds the mass of the axion, particle production from axion decays become ineffective. This puts a severe constraint on the mass of the dark photon; the simplest possibility is that the dark photon is massless. We also assume that the dark photon is in its vacuum state, with no light matter charged under it in the universe, when the axion oscillations start around the QCD phase transition. If there is a non-negligible abundance of matter charged under the dark photon it generates a Debye mass for the dark photon, shutting off particle production. The presence of this Debye mass is precisely why the axion coupling to the photon cannot be utilized for our mechanism. The existence of light degrees of freedom charged under the dark photon would also lead to large self-interaction for the dark photons which would also suppress the particle production effect. Thus, we assume that matter charged under the dark photon is heavy, which is the natural expectation if it is a scalar or a vectorlike fermion.

\subsection{The equations of motion}

The equation of motion (EOM) for the homogeneous axion field in the FriedmannRobertson-Walker cosmological background with the scale factor $a(\eta)$ is written as

$$
\phi^{\prime \prime}+2 a H \phi^{\prime}+a^{2} \frac{\partial V}{\partial \phi}=\frac{a^{2}}{4 f_{d}}\left(F_{D}\right)_{\mu \nu} \tilde{F}_{D}^{\mu \nu}
$$


where the prime denotes derivative with respect to conformal time $\eta$ and we defined $1 / f_{d}=$ $\alpha_{d} c_{d} / 2 \pi f_{a}$. Working in Coulomb gauge, $\vec{\nabla} \cdot \vec{A}=0$, the equations of motion for the gauge fields are

$$
\begin{aligned}
A_{0} & =0 \\
\vec{A}^{\prime \prime}-\nabla^{2} \vec{A} & =-\frac{\phi^{\prime}}{f_{d}} \vec{\nabla} \times \vec{A}
\end{aligned}
$$

We treat the homogenous axion field as a classical field and focus on the production of gauge field quanta due to the coupling between the axion and gauge field. To see this particle production effect we decompose the gauge field in terms of creation and annihilation operators

$$
A(\eta, \vec{x})=\sum_{\lambda= \pm} \int \frac{d^{3} k}{(2 \pi)^{3}} a_{\lambda}^{\vec{k}} A_{\lambda}(\eta, \vec{k}) \epsilon_{\lambda} e^{i \vec{k} \cdot \vec{x}}+\text { h.c. }
$$

where $\lambda= \pm$ are the helicities of the gauge field. Plugging this decomposition into eq. (4.3) we find

$$
A_{ \pm}^{\prime \prime}+\left(k^{2} \pm \frac{k \phi^{\prime}}{f_{d}}\right) A_{ \pm}=0
$$

We fix the initial conditions for the gauge fields to be consistent with the Bunch-Davies vacuum

$$
A_{ \pm}\left(\eta \ll \eta_{\mathrm{QCD}}\right) \rightarrow \frac{e^{-i k \eta}}{\sqrt{2 k}}
$$

with $\eta_{\mathrm{QCD}}$ the conformal time of the QCD phase transition. The energy density in the gauge field is given by

$$
\rho_{d}=\frac{1}{2 a^{4}} \int \frac{d^{3} k}{(2 \pi)^{3}}\left(\left|\frac{\partial A_{+}}{\partial \eta}\right|^{2}+\left|\frac{\partial A_{-}}{\partial \eta}\right|^{2}+k^{2}\left(\left|A_{+}\right|^{2}+\left|A_{-}\right|^{2}\right)-2 k\right)
$$

where the last term comes from subtracting the vacuum energy, to render the integral finite. From this expression we see that if some modes of the gauge field described by eq. (4.3) experience a large growth this leads to an increase in the energy of the radiation field. This energy gain corresponds to particles being generated by the coupling to the time dependent classical axion field.

By energy conservation, the increase in energy of the radiation field leads to a decrease in energy of the axion. To study the backreaction effects of the particle production in the dynamics of the axion we will treat the right-hand side of eq. (4.1) as a mean field

$$
\left(F_{D}\right)_{\mu \nu} \tilde{F}_{D}^{\mu \nu} \rightarrow\left\langle\left(F_{D}\right)_{\mu \nu} \tilde{F}_{D}^{\mu \nu}\right\rangle=\frac{1}{a^{4}} \int \frac{d^{3} k}{(2 \pi)^{3}} \frac{|\vec{k}|}{2} \frac{\partial}{\partial \eta}\left(\left|A_{+}\right|^{2}-\left|A_{-}\right|^{2}\right)
$$




\subsection{Conditions for efficient particle production}

These coupled differential equations can be solved numerically to study the evolution of the axion. Before presenting our numerical results, we first discuss when we would expect particle production to be efficient. For this qualitative discussion we ignore the temperature dependence of the axion mass and the change in the number of relativistic degrees of freedom during its evolution. We include these effects for our numerical analysis in the next section.

For simplicity we approximate the axion potential by keeping only its leading quadratic term

$$
V(\phi) \approx \frac{1}{2} m_{a}^{2} \phi^{2}+\ldots
$$

which is a good approximation for $\phi \lesssim f_{a}$. The axion starts oscillating once Hubble drops below the axion mass

$$
\phi \approx \phi_{i} \cos \left(m_{a} t\right)\left(\frac{a_{i}}{a}\right)^{3 / 2}
$$

where $t$ is the comoving time and $a_{i}$ is the scale factor when $H=m_{a}$. This equation holds as long as the backreaction due to the gauge fields is small.

The fact the coupling in eq. (3.4) can lead to a very large production of the gauge field can be seen directly from the equations of motion for the gauge field

$$
A_{ \pm}^{\prime \prime}+\omega^{2}\left(k, \phi^{\prime}\right) A_{ \pm}=0, \quad \omega^{2}\left(k, \phi^{\prime}\right)=k^{2} \pm \frac{k \phi^{\prime}}{f_{d}} .
$$

If the effective frequency $\omega^{2}$ of a given mode becomes negative, the corresponding mode becomes tachyonic which can lead to an exponential growth of the corresponding "mode function". From eq. (4.11) one sees that the largest tachyonic frequency is

$$
\left|\omega_{t}\right| \lesssim \frac{\phi^{\prime}}{2 f_{d}} \approx \frac{a m_{a} \phi_{i}}{2 f_{d}}\left(\frac{a_{i}}{a}\right)^{3 / 2} \leq a m_{a} \frac{r \theta_{i}}{2}
$$

where we have used the dimensionless variable $\theta=\phi / f_{a}$, and recall that $r=f_{a} / f_{d}$. The largest tachyonic frequency occurs near the start of the oscillations, before the amplitude of $\phi$ decreases due to Hubble friction.

In order for the exponential growth to occur, another condition must be satisfied: the corresponding growth rate, $\sim|\omega|$, needs to be fast compared to both the Hubble ${ }^{1}$ expansion rate and to the time scale for which the effective frequency $\omega^{2}$ remains negative. When both conditions are satisfied, the growth of the mode $A(k)$ can be estimated using a WKB approximation

$$
A \propto \exp \left(\int d \tilde{\eta}|\omega|\right)
$$

where the $\tilde{\eta}$ integration is only over times in which the mode is tachyonic.

A given frequency mode remains tachyonic over a half-period of the axion, before $\phi^{\prime}$ changes sign. Thus, the conformal time interval in which a mode is tachyonic is approximately given by $\Delta \eta_{\text {tachyon }} \simeq 1 /\left(a m_{a}\right)$. There is no real growth of the gauge fields when

\footnotetext{
${ }^{1}$ Note that since we are using conformal time, one should compare the tachyon rate to $a H$ instead of just $H$.
} 
$|\omega| \ll\left(\Delta \eta_{\text {tachyon }}\right)^{-1}$. In this case, the time scale associated with the axion oscillation is too fast compared to the typical time scale for tachyon growth, and therefore the gauge field modes effectively respond to an averaged axion field, and averaging $\phi^{\prime}$ over an oscillation is almost zero. Thus, the lower bounds on the frequency that experiences an exponential production is

$$
\begin{aligned}
& \left|\omega_{t}\right| \gtrsim a H \\
& \left|\omega_{t}\right| \gtrsim\left(\Delta \eta_{\text {tachyon }}\right)^{-1} \approx a m_{a}
\end{aligned}
$$

The second condition is stronger than the first since $H \leq m_{a}$. Therefore, the gauge modes are exponentially produced in the following frequency band,

$$
a m_{a} \lesssim\left|\omega_{t}\right| \lesssim a m_{a} \frac{r \theta_{i}}{2}
$$

Given that $f_{a}$ determines the range for the axion field, the initial misalignment angle $\theta_{i}$ is bound to be at most $\mathcal{O}(1)$. Therefore, in order to have a significant production of gauge fields, one must have $f_{d}>f_{a}$. As we will show numerically in the next section, the ratio $f_{d} / f_{a}$ controls how effective the energy transfer between then axion condensate and the gauge field is.

In the relevant parameter space the gauge fields do not grow enough during the first oscillation of the axion field for them to influence its dynamics. At each oscillation the range of $k$-modes that experience tachyonic growth becomes smaller and eventually disappears. Therefore tachyonic particle production is only relevant during the first few oscillations of the axion. After a $k$-mode leaves the tachyonic frequency band it can still experience a significant growth sourced by the axion oscillations once its physical momenta redshifts enough for its frequency to enter the parametric resonance band. The growth due to the parametric resonant behavior is only relevant because it is Bose-enhanced due to the initial exponential growth of that mode during the tachyonic phase. The dynamics of the coupled axion-gauge field dynamics is very rich and due to its intrinsically non-linear nature it is very sensitive to variation of parameters and initial conditions as will be shown in the results section. Once all $k$-modes of the gauge field that experienced tachyonic growth redshift to momenta smaller than the axion mass, the axion-gauge field coupling becomes ineffective at depleting the axion energy, and the axion subsequently evolves as in the standard case.

\subsection{Dark photon contribution to $N_{\text {eff }}$}

The dark photons produced due to the tachyonic instability will carry a significant fraction of the energy density originally stored in the axion field. Since the gauge bosons are massless their energy density dilutes like radiation, and thus they contribute to the number of relativistic degrees of freedom $\left(N_{\text {eff }}\right)$ at $\mathrm{BBN}$ and also at the time of CMB decoupling. Their contribution to $N_{\text {eff }}$ is given by

$$
\Delta N_{\text {eff }}=\left.\frac{8}{7}\left(\frac{11}{4}\right)^{4 / 3} \frac{\rho_{d}}{\rho_{\gamma}}\right|_{T_{\gamma}=1 \mathrm{eV}}
$$

where $\rho_{\gamma}$ is the energy density in photons and $\rho_{d}$ is given by eq. (4.7). The contribution to $\Delta N_{\text {eff }}$ as a function of the parameters $f_{a}$ and $f_{d}$ can be computed using the numerical solutions to the gauge field-axion system, and will be presented in the next section. 
Assuming that most of the energy originally in the axion field is transferred to the dark photon we can obtain the approximate bound from $\Delta N_{\text {eff. }}$ As one can see from the numerical results in the next section, the transition between the gauge field feedback being negligible to it being very important in the axion evolution is very fast. We also find that this transition usually happens when the scale factor has increased (temperature has decreased) by an $\mathcal{O}(10)$ factor from when the oscillations started and that at this point an order one fraction of the axion energy is transferred to dark photons. Therefore, ignoring the temperature dependence of the mass (which is a very good approximation for $f_{a} \gtrsim 10^{17} \mathrm{GeV}$ ), we find

$$
\Delta N_{\mathrm{eff}} \sim \frac{g_{*} \pi^{2} \theta_{0}^{2} f_{a}^{2}}{90 M_{p}^{2}} \frac{a_{\mathrm{fb}}}{a_{\mathrm{osc}}},
$$

where $g_{*}$ is the number of relativistic degrees of freedom in the SM below the QCD phase transition and $a_{\mathrm{fb}}$ is the scale factor when the feedback due to the gauge fields becomes important for the axion. Using this simple approximation we see that for $f_{a} \gtrsim 10^{17} \mathrm{GeV}$ and $\theta_{0} \sim 1$, the contribution to $N_{\text {eff }}$ becomes order one and is thus in tension with observations. In the results section we present more precise bounds on $N_{\text {eff }}$ obtained from the numerical solutions.

\subsection{Numerical results}

In this section we present our numerical results. In order to produce these results we discretized the momentum integral in eq. (4.8). This was done by introducing a maximum momenta $k_{\text {cut }}=2 a_{i} m_{a} f_{a} / f_{d}$ and discretizing the interval $\left(0, k_{\text {cut }}\right)$ with over $10^{4}$ equally spaced points, and solving the coupled axion-gauge-field system until the particle production effect becomes negligible. We tested the stability of the code by varying the total number of discretized momenta, the maximum momenta $k_{\text {cut }}$ and the precision of the algorithm. For our numerical results we used the finite temperature potential of eq. (2.4) and included the temperature dependence of the number of relativistic degrees of freedom [6]. This leads to analogous, but quantitatively different conditions from eq. (4.15).

In figures 2 and 3 we show how particle production affects the evolution of the axion energy density. After an initial period where the axion abundance follows the standard axion, the dark photons backreact on the axion, damping its energy density by many orders of magnitude. We see that $f_{a} \simeq 10^{16} \mathrm{GeV}$ and $10^{17} \mathrm{GeV}$ are perfectly consistent with measurement of $N_{\text {eff }}$ by Planck [97]. Larger decay constants would produce too much dark radiation and hence are excluded. Future CMB experiments [98] aim to measure $\Delta N_{\text {eff }}$ to $10^{-2}$ level, which will be sensitive to decay constants of $f_{a} \gtrsim 10^{16} \mathrm{GeV}$. We note that these results are for an initial misalignment angle $\theta_{i}=1$, and the $N_{\text {eff }}$ constraints are ameliorated for a smaller initial misalignment angle.

In figure 4 we show that the depletion of the axion abundance is quite sensitive to initial conditions and parameters of the theory. This "chaotic" behavior likely arises from the rich dynamics of many coupled oscillators with time-dependent frequencies of the gauge field $k$-modes, which continually enter and exit a phase of parametric resonance with the axion. This chaotic behavior also appears in various preheating models which use broad 

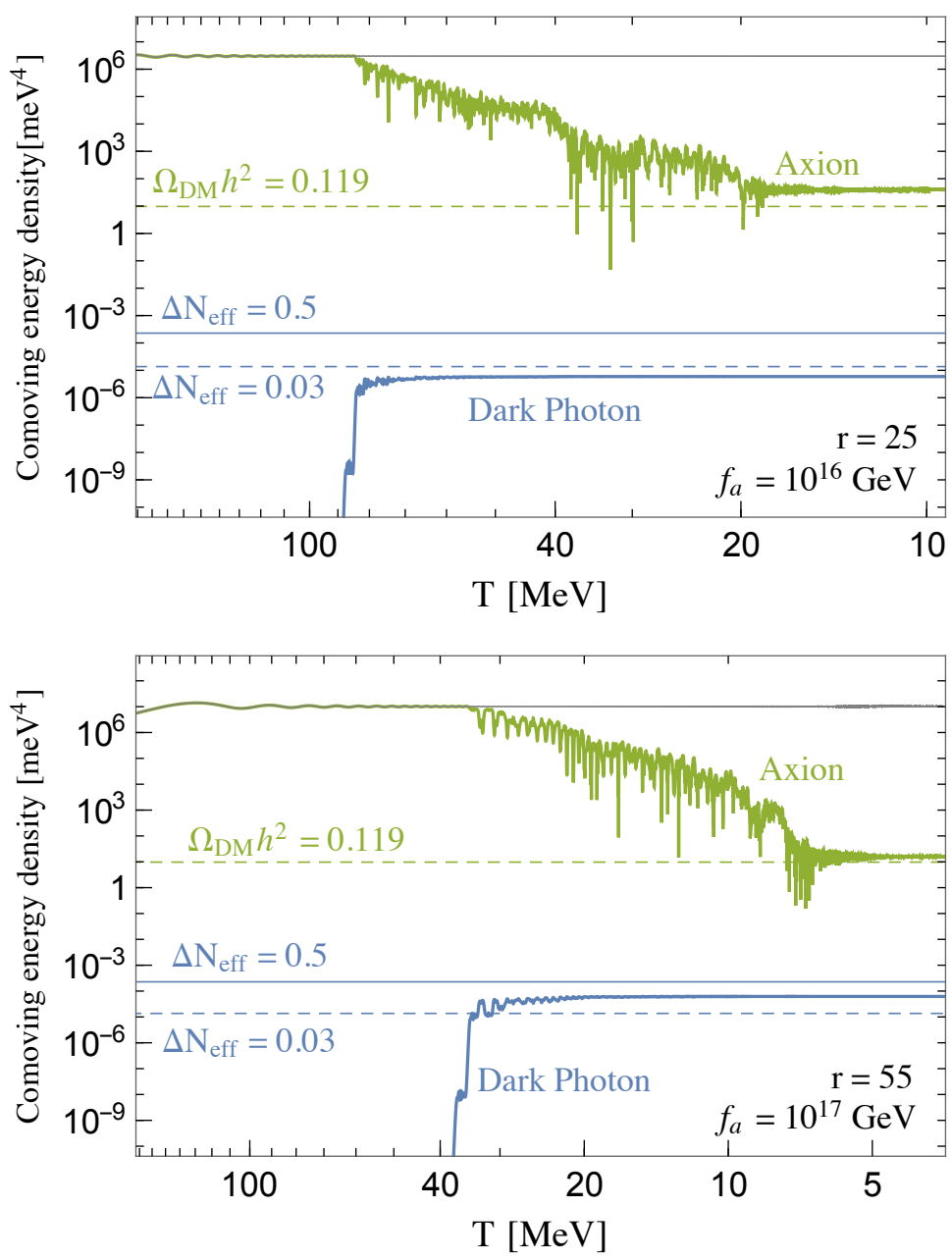

Figure 2. Comoving energy densities of the axion $\left(\rho_{a} a^{3}\right.$, green $)$ and the dark photon $\left(\rho_{d} a^{4}\right.$, blue $)$ as a function of the temperature, with $a=1$ today, for two example values of $f_{a}$ and $r$. We also show the evolution of axion energy density in the absence of our mechanism (gray). The green dashed line indicates the correct dark matter abundance, and the blue horizontal lines indicate the current (solid) and future (dashed) sensitivity to the dark photon contribution to $\Delta N_{\text {eff }}$. The absolute value of the axion and the dark photon energy densities are comparable at the point in which the axion energy density starts departing from the usual QCD axion behavior. The initial misalignment was chosen to be $\theta_{i}=1$.

parametric resonance [99]. Depending on the initial conditions, this sensitivity can greatly enhance the primordial fluctuations of the axion. This is one of effects that can lead to a significant enhancement of the matter power spectrum at small scales. It would be interesting to study how much of this sensitivity persists once the effects of the axion inhomogeneities are consistently included.

In our results we ignored the backscattering of dark photons into axions. This effect is naively very small, as one would conclude by estimating the $\gamma_{D} \gamma_{D} \rightarrow \phi \phi$ rate. However, due to the very large occupation number of dark photons the $2 \rightarrow 2$ estimate is not reliable. In fact, previous work which used a similar tachyonic production of gauge fields for preheating 

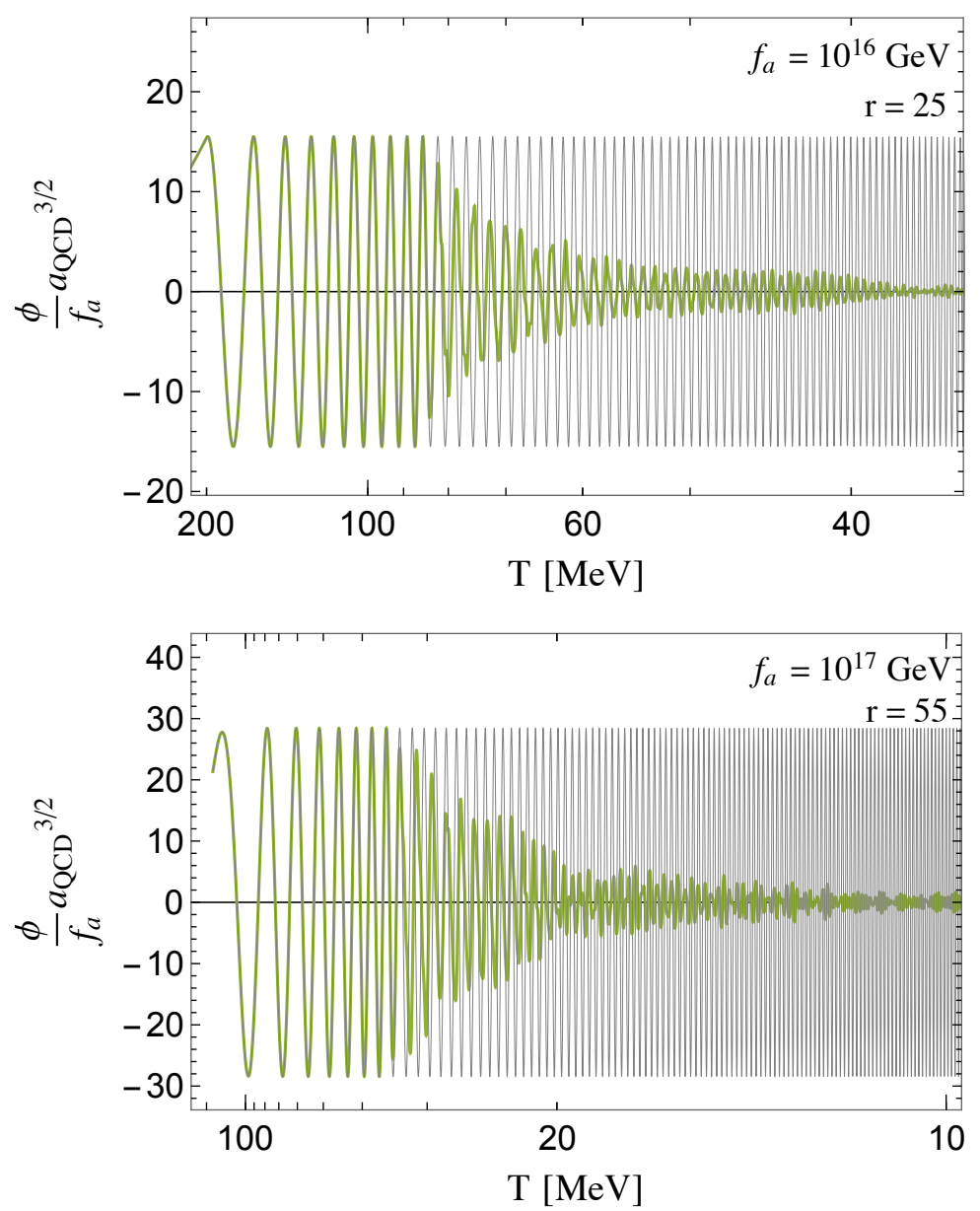

Figure 3. The decay of the comoving axion amplitude (green) as a function of temperature due to particle production for two example values of $f_{a}$ and $r$, compared with the axion amplitude in the absence of our mechanism (gray). We normalize the scale factor $a_{\mathrm{QCD}}(T) \equiv \frac{a(T)}{a(1 \mathrm{GeV})}$. The initial misalignment was chosen to be $\theta_{i}=1$.

and for generating primordial magnetic fields found that backscattering effects can lead to quantitative changes in the results $[46,100,101]$. Our preliminary investigations on the effects of backscattering indicate that in many cases this effect creates inhomogeneities in the axion field which can have energies comparable to the energy in the homogeneous component after the latter has started efficiently transferring energy to the gauge fields. However, the energy density in this inhomogeneous component of the axion is still negligible compared to the energy in the gauge fields. ${ }^{2}$ Therefore, even though a full treatment that

\footnotetext{
${ }^{2}$ After the first version of this work appeared on the Arxiv, ref. [102] presented the results of their numerical investigation which includes the backscattering effects of the gauge fields into axion perturbations. They claim that the energy in the axion perturbations can become comparable to the energy in the gauge fields, which reduces significantly the dilution of the energy in the axion condensate. Details of their calculation are relegated to a forthcoming publication. If true, this will significantly modify our results for higher values of $f_{a}$. This is an important question which requires further investigation, especially in light of a contrasting study by ref. [46] which studied the same effect in the context of preheating.
} 

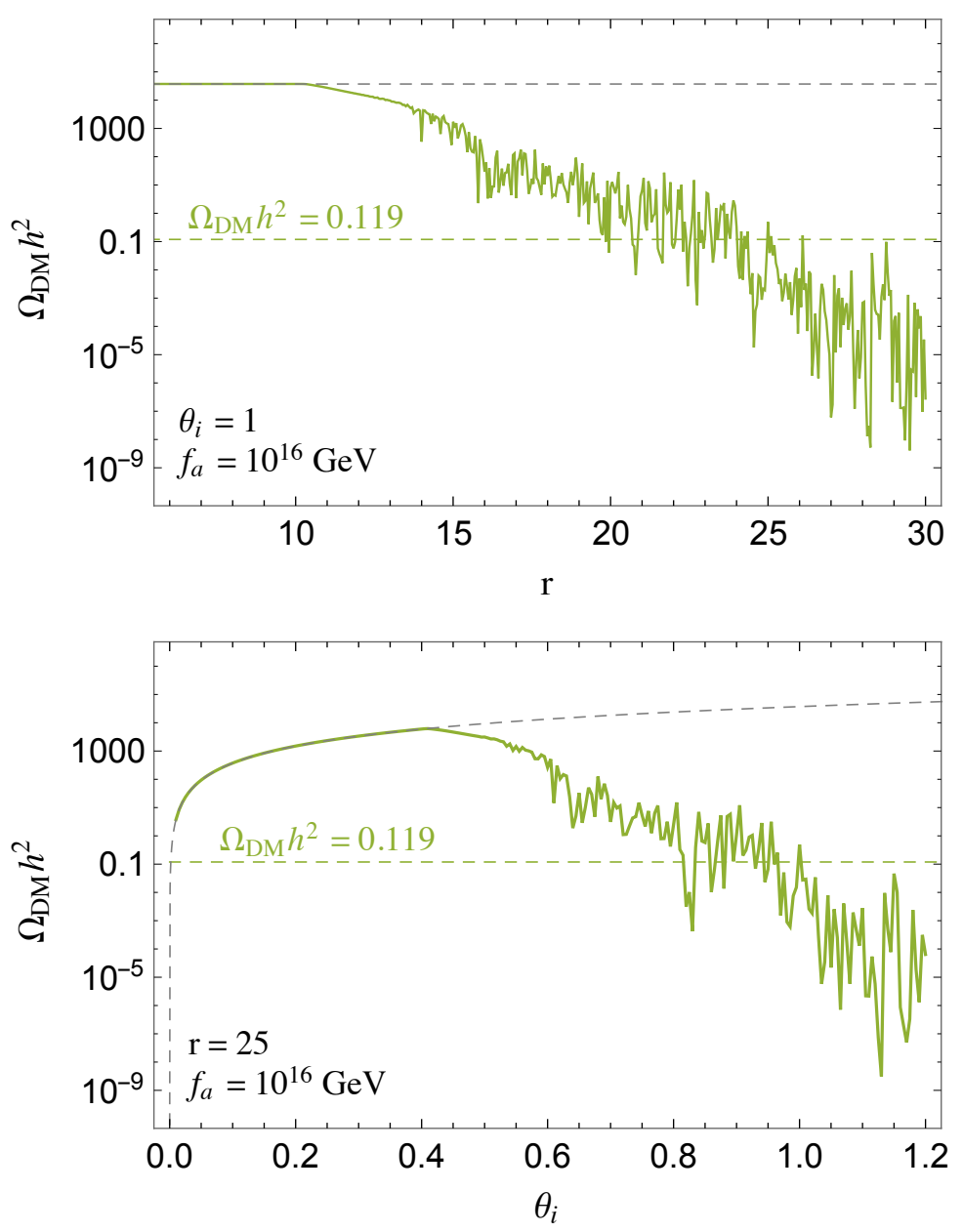

Figure 4. The sensitivity of the axion abundance to the ratio $r=f_{a} / f_{d}$ (left) and to the initial misalignment angle $\theta_{i}$ (right). The dashed gray line is the axion abundance in the absence of the dark photon coupling. We see that the mechanism is ineffective for small values of $r$ and $\theta_{i}$. For larger values of $\left\{r, \theta_{i}\right\}$, the depletion is larger and very sensitive to the initial conditions/parameters.

includes the effects of these perturbations might lead to quantitative changes in the final results, the qualitative picture of very large energy transfer from the axion condensate to the gauge fields is still expected to hold. A complete treatment of this effect could reveal very interesting features for axion DM. In particular, it might show that at small scales (comparable to the axion Compton wavelength) the dark matter density perturbations are much larger than the ones seeded by inflation, and thus lead to axion miniclusters, which are usually only associated with axion models with PQ symmetry breaking after inflation $[103,104]$.

\section{$5 \quad$ Future directions and conclusions}

The QCD axion is a well-motivated dark matter candidate, and there is a large experimental program for its search that is underway. There has been relatively little work on simple 
extensions of the QCD axion which can dramatically alter the cosmological evolution of the axion. We present one such extension where the axion has a coupling to a dark photon. This leads to an efficient depletion of the axion density into dark photons, such that large- $f$ QCD axions can be misaligned by a generic amount from their minimum. This makes the theoretically motivated large- $f$ parameter space viable.

For a region of parameter space, this additional energy density in dark photons can be seen in future CMB experiments. Given the QCD axion mass, an order of magnitude estimate of this energy density can be obtained (the prediction is uncertain due to the initial misalignment angle), and hence constitutes a test for this mechanism. This mechanism also relaxes the isocurvature bounds on axion DM. If future experiments measure the energy scale of inflation, they can rule out a large range of axion decay constants in anthropic models which would still be allowed in our scenario.

We have presented a minimal extension of the QCD axion model which focused only on the interactions of a dark photon with the axion. There are many extensions of this simple model, which include interactions of the dark photon with other SM fields that can lead to interesting predictions and are worth exploring. There might also be interactions of the axion with fields other than a dark photon that may give us a similar energy dissipation mechanism.

\section{Acknowledgments}

We thank Anson Hook, Curly Huang, Lisa Randall, Matt Reece, Ben Safdi and Jesse Thaler for discussions and for useful comments on our manuscript. We would like to thank Francis-Yan Cyr-Racine, Peter Graham, Mehrdad Mirbabayi, Konrad Tywoniuk, Yue Zhao for useful discussions. The work of PA is supported by the National Science Foundation (NSF) grants PHY-0855591 and PHY-1216270. The work of GMT is supported by the U.S. Department of Energy (DOE) grant DE-SC0012012 and the NSF Grant 1316699. The work of WX is supported by DOE under grant contract numbers DE-SC-00012567 and DE-SC-00015476.

\section{A UV completions of the alignment model}

In this appendix we complete the model presented in section 3 , focusing on generating the alignment potential term,

$$
V(a, b)=-\Lambda^{4} \cos \left(\frac{n a+b}{f}\right) .
$$

The purpose of this is to show the origin of large $n$ in the coupling. In this section, we will only focus on generating this potential term for the two axions. We do not explicitly write down the couplings of the axions to QCD/dark photons for clarity of presentation, but these can be easily included.

We note that the examples presented below have a large degree of overlap - we can use $4 \mathrm{D}$ confining dynamics in a 5D axion model, and all variants of the mechanisms can be "iterated" in a clockwork. 


\section{A.1 A 5D model}

We consider a model where axions arise from gauge fields in 5D [105, 106]. The 4D axions are identified with phases of Wilson loops around the extra dimension. The 5D model consists of two gauge fields in $5 \mathrm{D},\left(A_{M}, B_{M}\right)$. The fifth dimension is compactified on an orbifold $S_{1} / Z_{2}$ at a high scale, $\frac{1}{2 \pi R} \equiv \Lambda$, with boundary conditions such that $\left(A_{5}, B_{5}\right)$ zero modes survive at low energies [90, 91, 93]. These serve as the axions $(a, b)$. There is an additional fermion $\psi$ in $5 \mathrm{D}$ which has charges $(n, 1)$ under the two gauge groups.

$$
\mathcal{L}=\int d y \sqrt{G}\left[-\frac{1}{4 g^{2}} F_{A, \mu \nu} F_{A}^{\mu \nu}-\frac{1}{4 g^{2}} F_{B, \mu \nu} F_{B}^{\mu \nu}-\bar{\psi}(i \not D+m) \psi\right]
$$

Choosing different 5D gauge couplings for the two gauge groups does not by itself lead to a hierarchy in axion couplings, so for simplicity we have chosen them to be identical. If the mass of the fermion is smaller than the 5D Kaluza-Klein scale, it generates a sizeable Casimir potential for $\left(A_{5}, B_{5}\right)$. The $4 \mathrm{D}$ effective Lagrangian is

$$
\mathcal{L}=2 \pi R\left[\frac{1}{2 g^{2}} \partial_{\mu} A_{5} \partial^{\mu} A_{5}+\frac{1}{2 g^{2}} \partial_{\mu} B_{5} \partial^{\mu} B_{5}+\frac{3}{4 \pi^{2}(2 \pi R)^{5}} \cos \left(2 \pi R\left(n A_{5}+B_{5}\right)\right)\right]
$$

We identify $\sqrt{2 \pi R g^{2}}=1 / f$, such that the Lagrangian for canonically normalized fields $(a, b)$ is,

$$
\mathcal{L}=\frac{1}{2} \partial_{\mu} a \partial^{\mu} a+\frac{1}{2} \partial_{\mu} b \partial^{\mu} b+\frac{3 \Lambda^{4}}{4 \pi^{2}} \cos \left(\frac{n a+b}{f}\right) .
$$

We have neglected higher harmonics of the cosine potential and finite $\psi$-mass effects which are subdominant to this term, and do not affect the alignment qualitatively. We note that the coupling of the axions to QCD and the dark photon can be obtained in this model by including appropriate mixed Chern-Simons terms.

\section{A.2 Confining dynamics}

A mechanism along the lines of above can be obtained by a non-abelian gauge group $\operatorname{SU}(N)$ that confines at a high scale $\Lambda$. We assume $n$ flavors of fermions in the fundamental representation of $\mathrm{SU}(N)$ that are charged under the $\mathrm{U}(1)_{P Q, a}$ and a single $\mathrm{SU}(N)$ fundamental that is charged under the $\mathrm{U}(1)_{P Q, b}$.

\begin{tabular}{|c|ccc|}
\hline & $\mathrm{SU}(N)$ & $\mathrm{U}(1)_{P Q, a}$ & \\
\hline$Q$ & $N$ & 1 & \\
$Q^{c}$ & $\bar{N}$ & 1 & \\
$\Phi_{a}$ & 1 & 2 & \\
\hline
\end{tabular}

\begin{tabular}{|c|cc|}
\hline & $\mathrm{SU}(N)$ & $\mathrm{U}(1)_{P Q, b}$ \\
\hline$q$ & $N$ & 1 \\
$q^{c}$ & $\bar{N}$ & 1 \\
$\Phi_{b}$ & 0 & 2 \\
\hline
\end{tabular}

This is very similar to the $\operatorname{KSVZ}[63,64]$ model. When the $\mathrm{U}(1)_{P Q}$ 's are spontaneously broken at scale $f$, the fermions $Q, q$ get masses $\sim f$. The phases of $\Phi_{a, b}$ couple to the $\mathrm{SU}(N)$ field strength with the following anomaly coefficient,

$$
\mathcal{L}=\frac{\alpha}{2 \pi}\left(\frac{n a}{f}+\frac{b}{f}\right) G \tilde{G}
$$


where $\alpha$ and $G$ are the coupling and field strength for $\mathrm{SU}(N)$ respectively. This results in the axion potential

$$
V(a, b)=-\Lambda_{\mathrm{SU}(N)}^{4} \cos \left(\frac{n a+b}{f}\right) .
$$

We now demonstrate that we can obtain a large confinement scale for the $\mathrm{SU}(N)$ gauge group such that one mass eigenstate is heavy and decouples from the low-energy theory. We assume that the $\mathrm{SU}(N)$ gauge theory is weakly coupled at the scale $f$, such that the 't Hooft coupling $\lambda(f)=4 \pi \alpha(f) N \lesssim 1$. We also choose the $\mathrm{SU}(N)$ theory to be asymptotically free,

$$
b_{+}=-\frac{11}{3} N+\frac{2}{3}(n+1)<0
$$

such that the theory remains weakly coupled in the UV. Here $b_{+}$is the coefficient of the $\beta$-function for the gauge coupling above the scale $f$. The dimensionally transmuted scale is,

$$
\Lambda_{\mathrm{SU}(N)}=f \exp \left(\frac{2 \pi}{\alpha(f) b_{-}}\right)
$$

where $b_{-}=-\frac{11}{3} N$ is the coefficient of the IR $\beta$ function. We see that we can get a high confinement scale, which decouples the heavy axion.

Note that there are a large number of degrees of freedom in this model, $\mathcal{O}\left(n^{2}\right)$, which can renormalize the Planck scale to be too low. To ameliorate this, we can use the clockwork mechanism outlined below.

\section{A.3 Clockwork}

The models considered above can be extended to include more axions, such that the large charges encountered in those models are traded for a specific charge structure [88, 93-96].

Consider a model with $m+2$ scalars each of which get a vev $f$ which spontaneously breaks a $\mathrm{U}(1)$, resulting in $m+2$ axions. We add the following interaction between these scalars,

$$
\mathcal{L}=\kappa\left(\Phi_{b}^{\dagger} \phi_{1}^{q}+\phi_{1}^{\dagger} \phi_{2}^{q}+\ldots+\phi_{m}^{\dagger} \Phi_{a}^{q}\right)+\text { h.c. }
$$

These couplings explicitly break $\mathrm{U}(1)^{m+2} \rightarrow \mathrm{U}(1)$. The would-be Goldstones from spontaneous breaking of these symmetries are then massive and can be decoupled from the low-energy theory. The mass matrix for the Goldstones is

$$
\mathcal{M}=\kappa f^{q+1}\left[\left(b-q \pi_{1}\right)^{2}+\left(\pi_{m}-q a\right)^{2}+\sum_{i=1}^{m-1}\left(\pi_{i}-q \pi_{i+1}\right)^{2}\right]
$$

We have not included the contribution of QCD to the Goldstone masses, so there is one eigenstate of $\mathcal{M}$ with a zero eigenvalue. We are interested in the projection of the $a, b$ fields on to this eigenstate, $\phi$. It is easily seen that the normalized eigenvector with the zero eigenvalue is $\frac{1}{\mathcal{N}}\left\{1, \frac{1}{q}, \ldots \frac{1}{q^{m+1}}\right\}$, with

$$
\mathcal{N}=\sqrt{\frac{1-q^{-2(m+1)}}{1-q^{-2}}}
$$


which is $\mathcal{O}(1)$ for $q, m>1$. Thus,

$$
\begin{aligned}
& b=\frac{1}{\mathcal{N}} \phi+\ldots \\
& a=\frac{1}{\mathcal{N}} \frac{1}{q^{m+1}} \phi+\ldots
\end{aligned}
$$

Therefore we see that the effective hierarchy in charges that we get is $n=q^{m+1}$. For $q=3, m=4$, we easily get $n=\mathcal{O}(100)$.

Open Access. This article is distributed under the terms of the Creative Commons Attribution License (CC-BY 4.0), which permits any use, distribution and reproduction in any medium, provided the original author(s) and source are credited.

\section{References}

[1] P. Arias, D. Cadamuro, M. Goodsell, J. Jaeckel, J. Redondo and A. Ringwald, WISPy Cold Dark Matter, JCAP 06 (2012) 013 [arXiv:1201.5902] [INSPIRE].

[2] R.D. Peccei and H.R. Quinn, Some Aspects of Instantons, Nuovo Cim. A 41 (1977) 309 [INSPIRE].

[3] R.D. Peccei and H.R. Quinn, CP Conservation in the Presence of Instantons, Phys. Rev. Lett. 38 (1977) 1440 [INSPIRE].

[4] S. Weinberg, A New Light Boson?, Phys. Rev. Lett. 40 (1978) 223 [InSPIRE].

[5] F. Wilczek, Problem of Strong $p$ and $t$ Invariance in the Presence of Instantons, Phys. Rev. Lett. 40 (1978) 279 [INSPIRE].

[6] Particle Data Group collaboration, C. Patrignani et al., Review of Particle Physics, Chin. Phys. C 40 (2016) 100001 [inSPIRE].

[7] L.F. Abbott and P. Sikivie, A Cosmological Bound on the Invisible Axion, Phys. Lett. B 120 (1983) 133 [INSPIRE].

[8] M. Dine and W. Fischler, The Not So Harmless Axion, Phys. Lett. B 120 (1983) 137 [INSPIRE].

[9] J. Preskill, M.B. Wise and F. Wilczek, Cosmology of the Invisible Axion, Phys. Lett. B 120 (1983) 127 [INSPIRE].

[10] C. Vafa and E. Witten, Parity Conservation in QCD, Phys. Rev. Lett. 53 (1984) 535 [INSPIRE].

[11] C.A. Baker et al., An Improved experimental limit on the electric dipole moment of the neutron, Phys. Rev. Lett. 97 (2006) 131801 [hep-ex/0602020] [INSPIRE].

[12] B. Graner, Y. Chen, E.G. Lindahl and B.R. Heckel, Reduced Limit on the Permanent Electric Dipole Moment of ${ }^{199} \mathrm{Hg}$, Phys. Rev. Lett. 116 (2016) 161601 [arXiv:1601.04339] [INSPIRE].

[13] J.M. Pendlebury et al., Revised experimental upper limit on the electric dipole moment of the neutron, Phys. Rev. D 92 (2015) 092003 [arXiv: 1509.04411] [INSPIRE].

[14] CAST collaboration, S. Andriamonje et al., An Improved limit on the axion-photon coupling from the CAST experiment, JCAP 04 (2007) 010 [hep-ex/0702006] [INSPIRE]. 
[15] A. Ayala, I. Domínguez, M. Giannotti, A. Mirizzi and O. Straniero, Revisiting the bound on axion-photon coupling from Globular Clusters, Phys. Rev. Lett. 113 (2014) 191302 [arXiv: 1406.6053] [INSPIRE].

[16] H.E.S.S. collaboration, A. Abramowski et al., Constraints on axionlike particles with H.E.S.S. from the irregularity of the PKS 2155-304 energy spectrum, Phys. Rev. D 88 (2013) 102003 [arXiv: 1311.3148] [INSPIRE].

[17] Fermi-LAT collaboration, M. Ajello et al., Search for Spectral Irregularities due to Photon-Axionlike-Particle Oscillations with the Fermi Large Area Telescope, Phys. Rev. Lett. 116 (2016) 161101 [arXiv: 1603.06978] [INSPIRE].

[18] A. Payez, C. Evoli, T. Fischer, M. Giannotti, A. Mirizzi and A. Ringwald, Revisiting the SN1987A gamma-ray limit on ultralight axion-like particles, JCAP 02 (2015) 006 [arXiv: 1410.3747] [INSPIRE].

[19] ADMX collaboration, S.J. Asztalos et al., A SQUID-based microwave cavity search for dark-matter axions, Phys. Rev. Lett. 104 (2010) 041301 [arXiv:0910.5914] [INSPIRE].

[20] T.M. Shokair et al., Future Directions in the Microwave Cavity Search for Dark Matter Axions, Int. J. Mod. Phys. A 29 (2014) 1443004 [arXiv:1405.3685] [InSPIRE].

[21] E. Armengaud et al., Conceptual Design of the International Axion Observatory (IAXO), 2014 JINST 9 T05002 [arXiv: 1401.3233] [INSPIRE].

[22] A. Arvanitaki and A.A. Geraci, Resonantly Detecting Axion-Mediated Forces with Nuclear Magnetic Resonance, Phys. Rev. Lett. 113 (2014) 161801 [arXiv:1403.1290] [INSPIRE].

[23] B.M. Brubaker et al., First results from a microwave cavity axion search at $24 \mu \mathrm{eV}$, Phys. Rev. Lett. 118 (2017) 061302 [arXiv:1610.02580] [INSPIRE].

[24] T. Banks, M. Dine, P.J. Fox and E. Gorbatov, On the possibility of large axion decay constants, JCAP 06 (2003) 001 [hep-th/0303252] [INSPIRE].

[25] P. Svrček and E. Witten, Axions In String Theory, JHEP 06 (2006) 051 [hep-th/0605206] [INSPIRE].

[26] J.P. Conlon, The QCD axion and moduli stabilisation, JHEP 05 (2006) 078 [hep-th/0602233] [INSPIRE].

[27] D. Budker, P.W. Graham, M. Ledbetter, S. Rajendran and A. Sushkov, Proposal for a Cosmic Axion Spin Precession Experiment (CASPEr), Phys. Rev. X 4 (2014) 021030 [arXiv:1306.6089] [INSPIRE].

[28] A. Arvanitaki, M. Baryakhtar and X. Huang, Discovering the QCD Axion with Black Holes and Gravitational Waves, Phys. Rev. D 91 (2015) 084011 [arXiv:1411.2263] [INSPIRE].

[29] M. Silva-Feaver et al., Design Overview of DM Radio Pathfinder Experiment, IEEE Trans. Appl. Supercond. 27 (2016) 1400204 [arXiv:1610.09344] [INSPIRE].

[30] Y. Kahn, B.R. Safdi and J. Thaler, Broadband and Resonant Approaches to Axion Dark Matter Detection, Phys. Rev. Lett. 117 (2016) 141801 [arXiv:1602.01086] [InSPIRE].

[31] A. Arvanitaki, M. Baryakhtar, S. Dimopoulos, S. Dubovsky and R. Lasenby, Black Hole Mergers and the QCD Axion at Advanced LIGO, Phys. Rev. D 95 (2017) 043001 [arXiv: 1604. 03958] [INSPIRE].

[32] A. Garcon et al., The Cosmic Axion Spin Precession Experiment (CASPEr): a dark-matter search with nuclear magnetic resonance, arXiv: 1707.05312 [INSPIRE]. 
[33] B. Ratra, Cosmological 'seed' magnetic field from inflation, Astrophys. J. 391 (1992) L1 [INSPIRE].

[34] S.M. Carroll and G.B. Field, The Einstein equivalence principle and the polarization of radio galaxies, Phys. Rev. D 43 (1991) 3789 [INSPIRE].

[35] W.D. Garretson, G.B. Field and S.M. Carroll, Primordial magnetic fields from pseudoGoldstone bosons, Phys. Rev. D 46 (1992) 5346 [hep-ph/9209238] [INSPIRE].

[36] G.B. Field and S.M. Carroll, Cosmological magnetic fields from primordial helicity, Phys. Rev. D 62 (2000) 103008 [astro-ph/9811206] [INSPIRE].

[37] D.-S. Lee, W.-l. Lee and K.-W. Ng, Primordial magnetic fields from dark energy, Phys. Lett. B 542 (2002) 1 [astro-ph/0109184] [INSPIRE].

[38] L. Campanelli and M. Giannotti, Magnetic helicity generation from the cosmic axion field, Phys. Rev. D 72 (2005) 123001 [astro-ph/0508653] [InSPIRE].

[39] M.M. Anber and L. Sorbo, N-flationary magnetic fields, JCAP 10 (2006) 018 [astro-ph/0606534] [INSPIRE].

[40] M.M. Anber and L. Sorbo, Naturally inflating on steep potentials through electromagnetic dissipation, Phys. Rev. D 81 (2010) 043534 [arXiv:0908.4089] [INSPIRE].

[41] N. Barnaby and M. Peloso, Large NonGaussianity in Axion Inflation, Phys. Rev. Lett. 106 (2011) 181301 [arXiv:1011.1500] [INSPIRE].

[42] N. Barnaby, R. Namba and M. Peloso, Phenomenology of a Pseudo-Scalar Inflaton: Naturally Large NonGaussianity, JCAP 04 (2011) 009 [arXiv:1102.4333] [INSPIRE].

[43] N. Barnaby, E. Pajer and M. Peloso, Gauge Field Production in Axion Inflation: Consequences for Monodromy, non-Gaussianity in the CMB and Gravitational Waves at Interferometers, Phys. Rev. D 85 (2012) 023525 [arXiv:1110.3327] [INSPIRE].

[44] N. Barnaby, R. Namba and M. Peloso, Observable non-Gaussianity from gauge field production in slow roll inflation and a challenging connection with magnetogenesis, Phys. Rev. D 85 (2012) 123523 [arXiv: 1202.1469] [INSPIRE].

[45] P. Adshead, E. Martinec and M. Wyman, Gauge fields and inflation: Chiral gravitational waves, fluctuations and the Lyth bound, Phys. Rev. D 88 (2013) 021302 [arXiv:1301.2598] [INSPIRE].

[46] P. Adshead, J.T. Giblin, T.R. Scully and E.I. Sfakianakis, Gauge-preheating and the end of axion inflation, JCAP 12 (2015) 034 [arXiv:1502.06506] [INSPIRE].

[47] A. Notari and K. Tywoniuk, Dissipative Axial Inflation, JCAP 12 (2016) 038 [arXiv:1608.06223] [INSPIRE].

[48] J.T. Giblin, G. Kane, E. Nesbit, S. Watson and Y. Zhao, Was the Universe Actually Radiation Dominated Prior to Nucleosynthesis?, Phys. Rev. D 96 (2017) 043525 [arXiv:1706.08536] [INSPIRE].

[49] A. Hook and G. Marques-Tavares, Relaxation from particle production, JHEP 12 (2016) 101 [arXiv:1607.01786] [INSPIRE].

[50] A.D. Linde, Inflation and Axion Cosmology, Phys. Lett. B 201 (1988) 437 [InSPIRE].

[51] M. Tegmark, A. Aguirre, M. Rees and F. Wilczek, Dimensionless constants, cosmology and other dark matters, Phys. Rev. D 73 (2006) 023505 [astro-ph/0511774] [INSPIRE]. 
[52] P.J. Steinhardt and M.S. Turner, Saving the Invisible Axion, Phys. Lett. B 129 (1983) 51 [INSPIRE].

[53] G. Lazarides, R.K. Schaefer, D. Seckel and Q. Shafi, Dilution of Cosmological Axions by Entropy Production, Nucl. Phys. B 346 (1990) 193 [inSPIRE].

[54] M. Kawasaki, T. Moroi and T. Yanagida, Can decaying particles raise the upper bound on the Peccei-Quinn scale?, Phys. Lett. B 383 (1996) 313 [hep-ph/9510461] [INSPIRE].

[55] G.R. Dvali, Removing the cosmological bound on the axion scale, hep-ph/9505253 [INSPIRE].

[56] K. Choi, H.B. Kim and J.E. Kim, Axion cosmology with a stronger QCD in the early universe, Nucl. Phys. B 490 (1997) 349 [hep-ph/9606372] [INSPIRE].

[57] T. Banks and M. Dine, The Cosmology of string theoretic axions, Nucl. Phys. B 505 (1997) 445 [hep-th/9608197] [INSPIRE].

[58] T. Banks, M. Dine and M. Graesser, Supersymmetry, axions and cosmology, Phys. Rev. D 68 (2003) 075011 [hep-ph/0210256] [INSPIRE].

[59] A. Arvanitaki, S. Dimopoulos, S. Dubovsky, N. Kaloper and J. March-Russell, String Axiverse, Phys. Rev. D 81 (2010) 123530 [arXiv:0905.4720] [INSPIRE].

[60] Planck collaboration, P.A.R. Ade et al., Planck 2015 results. XX. Constraints on inflation, Astron. Astrophys. 594 (2016) A20 [arXiv:1502.02114] [INSPIRE].

[61] M. Kleban, M. Mirbabayi and M. Porrati, Effective Planck Mass and the Scale of Inflation, JCAP 01 (2016) 017 [arXiv: 1508. 01527] [INSPIRE].

[62] G. Grilli di Cortona, E. Hardy, J. Pardo Vega and G. Villadoro, The QCD axion, precisely, JHEP 01 (2016) 034 [arXiv:1511.02867] [INSPIRE].

[63] J.E. Kim, Weak Interaction Singlet and Strong CP Invariance, Phys. Rev. Lett. 43 (1979) 103 [INSPIRE].

[64] M.A. Shifman, A.I. Vainshtein and V.I. Zakharov, Can Confinement Ensure Natural CP Invariance of Strong Interactions?, Nucl. Phys. B 166 (1980) 493 [INSPIRE].

[65] E. Berkowitz, M.I. Buchoff and E. Rinaldi, Lattice QCD input for axion cosmology, Phys. Rev. D 92 (2015) 034507 [arXiv: 1505.07455] [INSPIRE].

[66] S. Borsányi et al., Axion cosmology, lattice QCD and the dilute instanton gas, Phys. Lett. B 752 (2016) 175 [arXiv: 1508.06917] [INSPIRE].

[67] C. Bonati et al., Axion phenomenology and $\theta$-dependence from $N_{f}=2+1$ lattice QCD, JHEP 03 (2016) 155 [arXiv: 1512.06746] [INSPIRE].

[68] J. Frison, R. Kitano, H. Matsufuru, S. Mori and N. Yamada, Topological susceptibility at high temperature on the lattice, JHEP 09 (2016) 021 [arXiv:1606.07175] [INSPIRE].

[69] S. Borsányi et al., Calculation of the axion mass based on high-temperature lattice quantum chromodynamics, Nature 539 (2016) 69 [arXiv:1606.07494] [INSPIRE].

[70] M. Dine, P. Draper, L. Stephenson-Haskins and D. Xu, Axions, Instantons and the Lattice, Phys. Rev. D 96 (2017) 095001 [arXiv:1705.00676] [INSPIRE].

[71] P. Fox, A. Pierce and S.D. Thomas, Probing a QCD string axion with precision cosmological measurements, hep-th/0409059 [INSPIRE]. 
[72] M.P. Hertzberg, M. Tegmark and F. Wilczek, Axion Cosmology and the Energy Scale of Inflation, Phys. Rev. D 78 (2008) 083507 [arXiv:0807.1726] [INSPIRE].

[73] E. Masso, F. Rota and G. Zsembinszki, On axion thermalization in the early universe, Phys. Rev. D 66 (2002) 023004 [hep-ph/0203221] [INSPIRE].

[74] P. Graf and F.D. Steffen, Thermal axion production in the primordial quark-gluon plasma, Phys. Rev. D 83 (2011) 075011 [arXiv: 1008.4528] [InSPIRE].

[75] A. Salvio, A. Strumia and W. Xue, Thermal axion production, JCAP 01 (2014) 011 [arXiv:1310.6982] [INSPIRE].

[76] L. Visinelli and P. Gondolo, Axion cold dark matter in non-standard cosmologies, Phys. Rev. D 81 (2010) 063508 [arXiv:0912.0015] [INSPIRE].

[77] R.T. Co, F. D'Eramo and L.J. Hall, Supersymmetric axion grand unified theories and their predictions, Phys. Rev. D 94 (2016) 075001 [arXiv:1603.04439] [InSPIRE].

[78] M. Kawasaki, F. Takahashi and M. Yamada, Suppressing the QCD Axion Abundance by Hidden Monopoles, Phys. Lett. B 753 (2016) 677 [arXiv:1511.05030] [InSPIRE].

[79] Y. Nomura, S. Rajendran and F. Sanches, Axion Isocurvature and Magnetic Monopoles, Phys. Rev. Lett. 116 (2016) 141803 [arXiv:1511.06347] [InSPIRE].

[80] J. Kearney, N. Orlofsky and A. Pierce, High-Scale Axions without Isocurvature from Inflationary Dynamics, Phys. Rev. D 93 (2016) 095026 [arXiv:1601.03049] [InSPIRE].

[81] M. Berg et al., Constraints on Axion-Like Particles from X-ray Observations of NGC1275, Astrophys. J. 847 (2017) 101 [arXiv: 1605.01043] [INSPIRE].

[82] M.C.D. Marsh, H.R. Russell, A.C. Fabian, B.P. McNamara, P. Nulsen and C.S. Reynolds, A New Bound on Axion-Like Particles, JCAP 12 (2017) 036 [arXiv:1703.07354] [InSPIRE].

[83] J.P. Conlon, F. Day, N. Jennings, S. Krippendorf and M. Rummel, Constraints on Axion-Like Particles from Non-Observation of Spectral Modulations for X-ray Point Sources, JCAP 07 (2017) 005 [arXiv: 1704.05256] [INSPIRE].

[84] R. Bähre et al., Any light particle search II - Technical Design Report, 2013 JINST 8 T09001 [arXiv: 1302.5647] [INSPIRE].

[85] K. Kaneta, H.-S. Lee and S. Yun, Portal Connecting Dark Photons and Axions, Phys. Rev. Lett. 118 (2017) 101802 [arXiv:1611.01466] [INSPIRE].

[86] K. Kaneta, H.-S. Lee and S. Yun, Dark photon relic dark matter production through the dark axion portal, Phys. Rev. D 95 (2017) 115032 [arXiv:1704.07542] [INSPIRE].

[87] J.E. Kim, H.P. Nilles and M. Peloso, Completing natural inflation, JCAP 01 (2005) 005 [hep-ph/0409138] [INSPIRE].

[88] G. Dvali, Black Holes and Large N Species Solution to the Hierarchy Problem, Fortsch. Phys. 58 (2010) 528 [arXiv:0706.2050] [InSPIRE].

[89] K. Choi, H. Kim and S. Yun, Natural inflation with multiple sub-Planckian axions, Phys. Rev. D 90 (2014) 023545 [arXiv: 1404.6209] [InSPIRE].

[90] Y. Bai and B.A. Stefanek, Natural millicharged inflation, Phys. Rev. D 91 (2015) 096012 [arXiv: 1405.6720] [INSPIRE].

[91] A. de la Fuente, P. Saraswat and R. Sundrum, Natural Inflation and Quantum Gravity, Phys. Rev. Lett. 114 (2015) 151303 [arXiv:1412.3457] [INSPIRE]. 
[92] K. Choi and S.H. Im, Realizing the relaxion from multiple axions and its UV completion with high scale supersymmetry, JHEP 01 (2016) 149 [arXiv:1511.00132] [INSPIRE].

[93] D.E. Kaplan and R. Rattazzi, Large field excursions and approximate discrete symmetries from a clockwork axion, Phys. Rev. D 93 (2016) 085007 [arXiv:1511.01827] [INSPIRE].

[94] G.F. Giudice and M. McCullough, A Clockwork Theory, JHEP 02 (2017) 036 [arXiv: 1610.07962] [INSPIRE].

[95] M. Farina, D. Pappadopulo, F. Rompineve and A. Tesi, The photo-philic QCD axion, JHEP 01 (2017) 095 [arXiv: 1611.09855] [INSPIRE].

[96] N. Craig, I. Garcia Garcia and D. Sutherland, Disassembling the Clockwork Mechanism, JHEP 10 (2017) 018 [arXiv:1704.07831] [INSPIRE].

[97] Planck collaboration, P.A.R. Ade et al., Planck 2015 results. XIII. Cosmological parameters, Astron. Astrophys. 594 (2016) A13 [arXiv:1502.01589] [INSPIRE].

[98] CMB-S4 collaboration, K.N. Abazajian et al., CMB-S4 Science Book, First Edition, arXiv: 1610.02743 [INSPIRE].

[99] L. Kofman, A.D. Linde and A.A. Starobinsky, Towards the theory of reheating after inflation, Phys. Rev. D 56 (1997) 3258 [hep-ph/9704452] [INSPIRE].

[100] P. Adshead, J.T. Giblin, T.R. Scully and E.I. Sfakianakis, Magnetogenesis from axion inflation, JCAP 10 (2016) 039 [arXiv:1606.08474] [INSPIRE].

[101] M. Peloso, L. Sorbo and C. Unal, Rolling axions during inflation: perturbativity and signatures, JCAP 09 (2016) 001 [arXiv:1606.00459] [INSPIRE].

[102] N. Kitajima, T. Sekiguchi and F. Takahashi, Cosmological abundance of the QCD axion coupled to hidden photons, arXiv:1711.06590 [INSPIRE].

[103] C.J. Hogan and M.J. Rees, Axion miniclusters, Phys. Lett. B 205 (1988) 228 [inSPIRE].

[104] E.W. Kolb and I.I. Tkachev, Large amplitude isothermal fluctuations and high density dark matter clumps, Phys. Rev. D 50 (1994) 769 [astro-ph/9403011] [INSPIRE].

[105] N. Arkani-Hamed, H.-C. Cheng, P. Creminelli and L. Randall, Extra natural inflation, Phys. Rev. Lett. 90 (2003) 221302 [hep-th/0301218] [INSPIRE].

[106] K.-w. Choi, A QCD axion from higher dimensional gauge field, Phys. Rev. Lett. 92 (2004) 101602 [hep-ph/0308024] [INSPIRE]. 